\title{
Morphometric and molecular study of corpus cavernosum of the rat after long- term high-fat diet intake.
}

\author{
I. Tomada*, N. Tomada**, H. Almeida*, P. Vendeira** and D. Neves* \\ * Laboratory of Molecular Cell Biology of Faculty of Medicine and IBMC of Universidade do \\ Porto, Alameda Professor Hernâni Monteiro, 4200-319 Porto, Portugal \\ ** Department of Urology of S. João Central Hospital, Alameda Professor Hernâni Monteiro, 4200- \\ 319 Porto, Portugal \\ inestomada@gmail.com
}

Erectile dysfunction (ED) is considered equivalent to endothelial dysfunction [1] and thus an early manifestation of atherosclerosis and cardiovascular disease (CVD) [2]. Atherosclerosis that is frequently provoked by regular consumption of high-fat diet (HFD) has been recently recognized as a chronic inflammation condition associated to vessel lipid deposition and vascular smooth cells proliferation that together impair endothelium-dependent relaxation of corpus cavernosum (CC) smooth muscle. It is also well established that HFD regular intake by its own induces endothelial dysfunction [3]. The main objective of this study was to evaluate effects of HFD continuous intake on atherosclerosis progression of $\mathrm{CC}$ vessels of the rat. Due to their intervention on atherosclerosisrelated vascular remodelling pathways, angiopoietins (Ang1, Ang2) expression in erectile tissue of this rat experimental model was characterized [4].

Thirty-six male Wistar rats (2 months-old) obtained from the colony of the IBMC of Universidade do Porto, were treated with high-fat diet (HFD) (TestDiet ${ }^{\circledR} \# 58 \mathrm{~V} 8,45 \%$ energy from fat;

metabolizable energy 4,65Kcal/g) until complete 6 months-old (HFD group), and 12 of them were submitted to caloric restriction (60\% of their usual caloric intake currently with maintenance diet) from 4 to 6 months-old (CR group). Age-matched control group rats ( $\mathrm{C}$ group) were fed with standard diet (Panlab® A04, 4\% energy from fat; metabolizable energy 2,90Kcal/g). Metabolic and biometric parameters were evaluated as previously described [5]. Rats were sacrificed by decapitation at 4 or 6 months. Penile fragments were removed, fixed in $10 \%$ buffered formaldehyde for $24 \mathrm{~h}$ and embedded in paraffin, oriented along its transversal axis. Tissue sections (4-6 $\mu \mathrm{m}$ thick) were placed in $0,1 \%$ poly-L-lysine-covered microscopy slides for immunohistochemical (IH) analysis of alpha-actin, Ang1 and Ang2. Mouse monoclonal anti-alpha-actin (Chemicon) (diluted 1/500), and goat polyclonal anti-Ang1 and anti-Ang2 (Santa Cruz Biotechnology) (diluted 1:100) followed by an adequate biotinylated secondary antibody and streptavidin-peroxidase complex (Vectastain-Vector), were employed to IH essays. Sections were reacted with diaminobenzidine/peroxidase, and counterstained with hematoxylin. Results were observed in a Light Microscope (Nikon). Smooth muscle layer (SML) morphometric analysis was performed with ImageJ® software (Image Processing and Analysis in Java, NIH, Maryland, USA), and the ratio of areas of SML to the total area of the tissue were calculated in 20 fields per animal under analysis. All data are expressed as mean \pm SEM, and statistical significance was assumed at $P$-level $<0,05$. Statistical analysis was performed with SPSS® software.

IH analysis of alpha-actin evidenced cavernous smooth muscle restricted to perivascular areas in all experimental groups (Fig. 1). The morphometric study showed significant differences in SML area between 6 months HFD treated rats and age-matched controls (16,0\% vs 10,9\%, P=0,00), and also 
between them and $\mathrm{CR}$ group (16,0\% vs $11,8 \%, \mathrm{P}=0,00)$. Adipocytes were observed close to vascular spaces (Fig. 2), particularly abundant in 6 months HFD rats, and scarce in CR animals. IH study of Ang1 and Ang2 (Fig. 2 (a) and (b), respectively, reveals their expression in SML and also in vascular endothelium in all studied animals. No differences were observed between experimental groups.

The present study reinforces the importance of dietary modulation on vascular function, and suggests that the deleterious effects of HFD in rat cavernous tissue, such as vascular lipid deposition and SML augmented thickness could be reversed by caloric restriction. Further molecular studies are needed to elucidate Ang1 and Ang2 roles in atherosclerotic process reversion in CC of HFD fed rats.

\section{References}

[1] A. Guay, Endocrinol. Metab. Clin. N. Am. 36 (2007) 453.

[2] H. Solomon, J. Man, G. Jackson, Heart. 89 (2003) 251.

[3] K. Esposito, F. Guigliano, C. Di Palo, et al, JAMA 291 (2004) 2978.

[4] L. Eklund, B. Olsen, Exp. Cell Res. 312 (2006) 630.

[5] I. Tomada, N. Tomada, F. Marques, P. Vendeira, D. Neves, Microsc. Microanal. 14 (supp 3) (2008) 126.

This study was partially supported by Faculty of Nutrition and Food Sciences of Universidade do Porto.

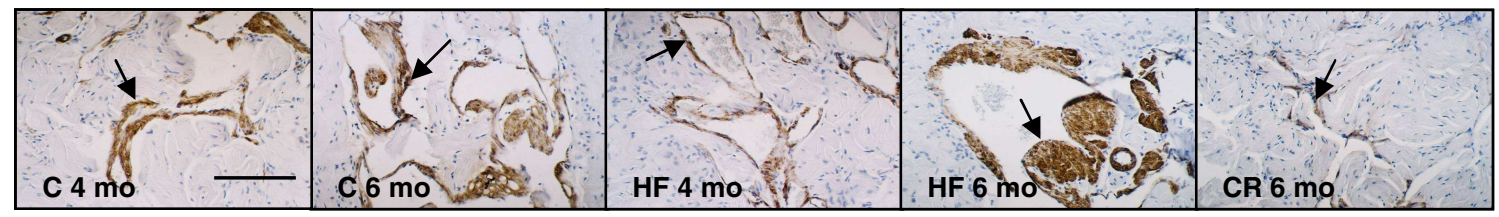

Fig. 1 - Alpha-actin immunohistochemical detection (arrow) in SML. Bar $50 \mu \mathrm{m}$.
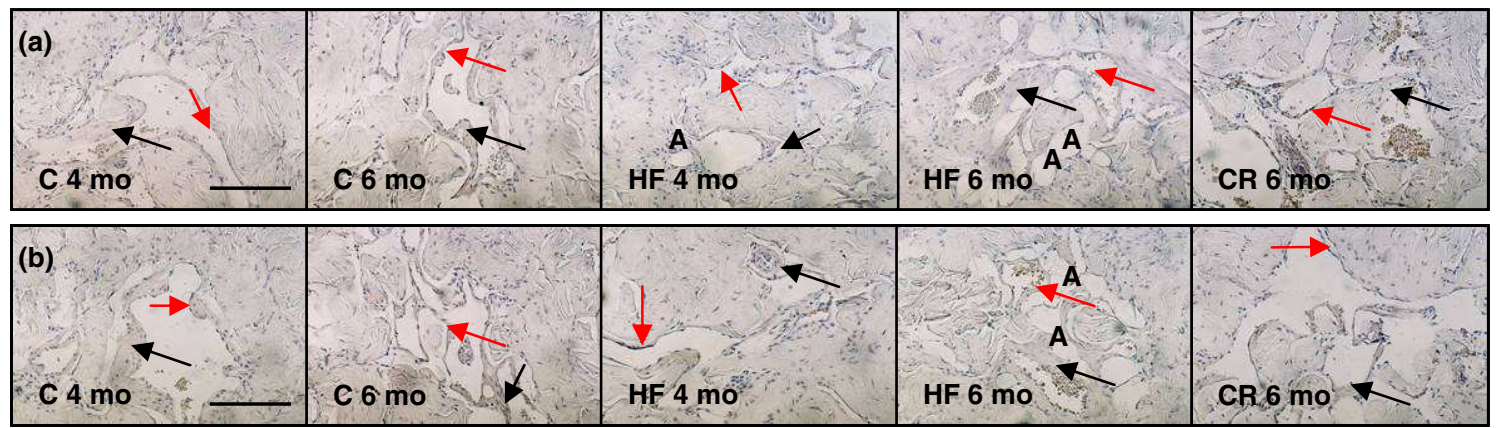

Fig. 2 - Ang1 (a) and Ang2 (b) immunohistochemical detection demonstrated their expression in smooth muscle (black arrows) and in endothelial cells (red arrows) in all studied animals. Adipocytes (A) were observed close to vascular spaces. Bar $50 \mu \mathrm{m}$. 